\title{
Commentary: Betting the trifecta: Gradient, prosthesis-patient mismatch, and durability
}

\author{
George L. Hicks, MD
}

From the University of Rochester Medical Center, Rochester, NY.

Disclosures: Author has nothing to disclose with regard to commercial support.

Received for publication Nov 6, 2018; accepted for publication Nov 6, 2018; available ahead of print Dec 5, 2018.

Address for reprints: George L. Hicks, MD, University of Rochester Medical Center, 601 Elmwood Ave, Roches-

ter, NY 14642 (E-mail: george_hicks@urmc.rochester.edu).

J Thorac Cardiovasc Surg 2019;158:1040

$0022-5223 / \$ 36.00$

Copyright (c) 2018 by The American Association for Thoracic Surgery

https://doi.org/10.1016/j.jtcvs.2018.11.012

In horse-betting parlance, the trifecta is a bet on 3 horses finishing win, place, or show in that order. It has more risk and therefore pays more to the bettor and remains a popular wager. The current article, "'Trifecta Has Lower Transvalvular Gradient and Less Prosthetic-Patient Mismatch Than Mosaic Ultra in the Aortic Position: A Prospective Randomized Trial," ${ }^{1}$ is a prospective single-institution analysis of 90 patients with aortic valvular pathology requiring aortic valve replacement. The purpose of the study is to compare size for size, 6-month hemodynamic performance of the Trifecta biological valve (St Jude Medical, St Paul, Minn) versus the Mosaic Ultra (Medtronic, Minneapolis, Minn).

The study was randomized at the time of surgery before annular sizing and 90 elective patients with small to medium annuli diameter (19-25 mm) after sizing with Hegar dilators were implanted with the Trifecta or the Mosaic Ultra aortic bioprostheses. All prostheses were placed in the supra-annular position using similar interrupted mattress suture techniques with postimplantation transesophageal echocardiography performed for valve and ventricular function, but not gradients measured because the authors believe that gradients are inaccurate at that time because of the type of valve, loading conditions, and varying ventricular function. Follow-up transthoracic echocardiograms were performed by blinded readers at 6 months. The simplified Bernoulli equation was used to calculate peak and mean pressure gradients across the prosthetic valve. Three consecutive cardiac cycles were recorded, 5 if atrial fibrillation was present. Peak gradient, mean pressure gradient, and indexed effective orifice area (EOAI) were calculated as the mean over these cycles. The authors found that both the peak and mean transvalvular gradients were approximately $50 \%$ lower in the Trifecta group compared with the Mosaic Ultra group for any given annulus size as measured by the metric Hegar sizer.

Furthermore, EAOI was significantly higher in the Trifecta group compared with the Mosaic Ultra group, with severe prosthesis-patient mismatch (EOAI $<0.65 \mathrm{~cm}^{2} / \mathrm{m}^{2}$ ) found in 10 of 36 patients $(28 \%)$ in the Mosaic group and 1 of 33 patients in the Trifecta group

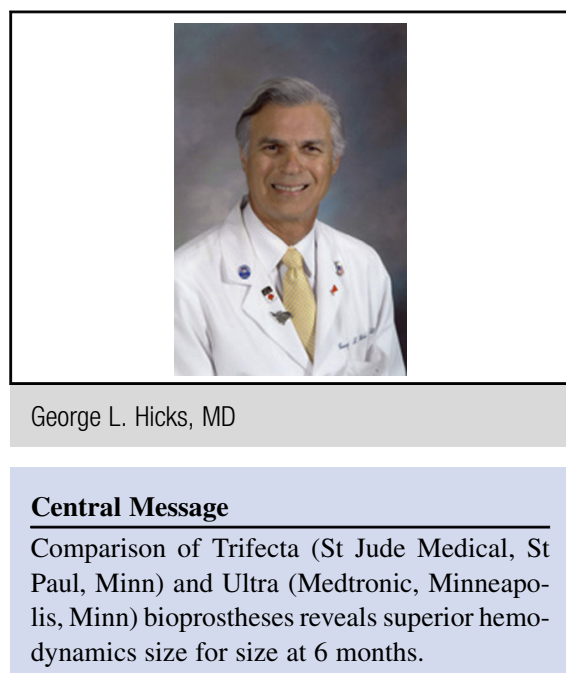

See Article page 1032.

(3\%). Moderate prosthesis-patient mismatch (EOAI $\leq 0.85 \mathrm{~cm}^{2} / \mathrm{m}^{2}$ ) was found in 33 of 36 patients in the Mosaic group $(92 \%)$ and in 12 of 33 patients in the Trifecta group $(36 \%)$. They found no difference between groups with regard to septal thickness.

The authors are to be congratulated on a randomized evaluation of 2 prostheses at 6 months after aortic valve replacement. Unfortunately, the study provides no guidance in terms of valve durability, patient activity status, left ventricular mass regression, or survival over the long term. Hopefully, this study will provide us details in the future on these important factors, and although studies at 6 to 7 years regarding the Trifecta are available (96\% freedom of structural change), a comparable evaluation of these 2 relatively equal patient cohorts could provide valuable long-term follow-up data.

Betting on horses to win the trifecta requires knowledge of the 3 best runners in the field. Betting on the Trifecta valve likewise requires objective long-term data regarding stability of low transvalvular gradient, lack of prosthesispatient mismatch, and valve durability with minimal structural deterioration. If future studies confirm these 3 factors in this bioprosthetic valve, then this horse could be a favorite to win money on the trifecta bet.

\section{Reference}

1. Braathen B, Husebye T, Lunde IG, Tønnessen T. Trifecta has lower gradient and less prosthesis-patient mismatch than Mosaic Ultra in the aortic position: a prospective randomized study. J Thorac Cardiovasc Surg. 2019;158:1032-9. 